\title{
Improving Backscatter Radio Tag Efficiency
}

\author{
Aggelos Bletsas, Member, IEEE, Antonis G. Dimitriou, Member, IEEE, and John N. Sahalos, Life Fellow, IEEE
}

\begin{abstract}
This paper studies tag properties for optimized tag-to-reader backscatter communication. The latter is exploited in RF identification (RFID) systems and utilizes binary reflection coefficient change of the tag antenna-load circuit. It is shown that amplitude maximization of complex reflection coefficient difference between the two states is not sufficient for optimized tag-to-reader backscatter communication, contrary to what is commonly believed in the field. We provide a general tag load selection methodology that applies to any tag antenna, including minimum scattering antennas as a special case. The method is based on tag antenna structural mode closed-form calculation (given three values of tag radar cross section), employs simple antenna/communication theory, and applies to both passive, as well as semipassive RFID tags.
\end{abstract}

Index Terms-Minimum scattering, receiver sensitivity, RF identification (RFID), structural mode.

\section{INTRODUCTION}

I MPRESSIVE progress has been observed since the first efforts on backscatter radio [1]. Numerous and diverse applications have emerged, spanning RF identifications (RFIDs) in electronic supply chain to electronic music instrumentation [2] or software-defined implementations for ultra-low-cost and ultra-low-power environmental sensor networks [3]-[5].

We focus on the tag-to-reader link and study the properties of the RFID tag for optimized tag-to-reader communication. Backscatter radio binary-modulates information with reflection coefficient change, when the tag antenna is alternatively connected between two different loads (Fig. 1). Work and results apply to passive, as well as semipassive (battery-assisted) RFID tags.

The specific contributions are summarized as follows.

- Necessary conditions (constraints) for improved tag-toreader backscatter communication are derived, while not restricting discussion to minimum scattering antennas or specific tag circuitry. It is shown that maximization of amplitude reflection coefficient difference is not sufficient; tag antenna structural mode should also be considered.

Manuscript received June 09, 2009, revised December 23, 2009; accepted March 18, 2010. Date of publication May 03, 2010; date of current version June 11, 2010. This work was implemented in the context of the Telecommunications Platform of Innovation Pole of C. M. Greece, supported by the General Secretariat Research and Technology and RFID in Healthcare supported by the Cyprus Research Promotion Foundation.

The authors are with the Radio-Communications Laboratory (RCL), Department of Physics, Aristotle University of Thessaloniki, Thessaloniki 54124, Greece, and also with the Telecom Laboratory, Electronic and Computer Engineering Department, Technical University of Crete, Crete 73100, Greece (e-mail: aggelos@telecom.tuc.gr; antodimi@auth.gr; sahalos@auth.gr).

Color versions of one or more of the figures in this paper are available online at http://ieeexplore.ieee.org.

Digital Object Identifier 10.1109/TMTT.2010.2047916
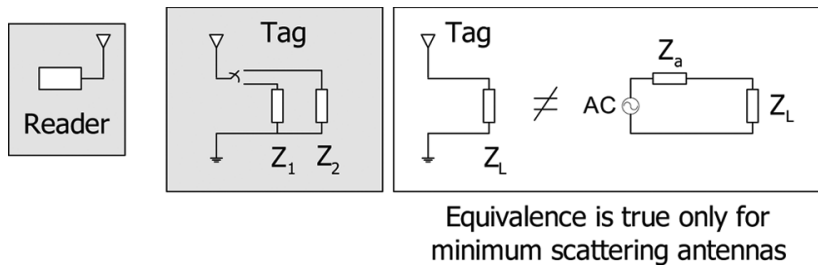

Fig. 1. Thevenin equivalent tag antenna-load circuit for the two terminating loads-states. Thevenin equivalent circuit is only valid for minimum scattering tag antennas. This work assumes no Thevenin modeling and studies the tag load selection problem for any tag antenna.

- Simple closed-form calculation of the tag antenna structural mode is provided. Proposed tag load selection for improved backscatter communication is based on the estimation of the tag antenna structural mode, applies to any tag antenna (including minimum scattering as special case), and covers both passive, as well as semipassive, tags.

Section II provides in more detail the connection of this work to prior art. Section III describes the constraints that the tag designer should adopt, utilizing communication theory, as well as the tag antenna structural mode. A method to calculate the tag antenna structural mode in closed form is provided in the Appendix . Section IV provides load selection design examples for both passive and semipassive tags. Finally, a conclusion is provided in Section V.

\section{CONNECTION TO PRIOR ART}

The ratio of power $P_{\text {out }}$ backscattered from tag to reader and power $P_{\text {ind }}$ induced at the tag defines the tag efficiency $K$ (e.g., $[6$, eq. (6)])

$$
\begin{aligned}
G K & \triangleq \frac{P_{\text {out }}}{P_{\text {ind }}}=\frac{S \sigma}{S A_{\text {eff }}} \Rightarrow \\
\sigma & =G K A_{\text {eff }}=\frac{\lambda^{2} G^{2}}{4 \pi} K
\end{aligned}
$$

where $A_{\text {eff }}, G$ is the tag antenna effective area and gain, respectively, $\lambda$ is the carrier wavelength, $S$ is the induced power density (in $\mathrm{W} / \mathrm{m}^{2}$ ) at the tag location from the field transmitted by the reader, and $\sigma$ is the tag antenna radar cross section (RCS).

RCS depends on the load connected to the tag antenna. Assume that $Z_{1}$ is the load connected to the tag antenna when the tag information bit is " 0 " and $Z_{2}$ is the load when the tag information bit is "1" (Fig. 1). Also denote $\vec{E}_{i}$ the (complex) backscattered field from the tag antenna when the latter is terminated at $Z_{i}$ ( $i=1$ for bit " 0 " or $i=2$ for bit "1"). Consecutively, RCS $\sigma_{i}$ is given [7], [8] by

$$
\sigma_{i} \triangleq \lim _{r \rightarrow+\infty} 4 \pi r^{2} \frac{\left|\vec{E}_{i}\right|^{2}}{\left|\vec{E}_{\text {ind }}\right|^{2}}=\frac{\lambda^{2}}{4 \pi} G^{2}\left|\Gamma_{i}-A_{s}\right|^{2}
$$


where $\sigma_{i}$ corresponds to tag load $Z_{i}(i=1$ or $i=2), Z_{a}$ is the tag antenna impedance, and $A_{s}$ corresponds to the structural mode of the tag antenna, which is a load-independent antenna quantity [8]. Its value is complex, in general, and depends on the geometry and materials used for the antenna construction. Furthermore,

$$
\Gamma_{i} \triangleq \frac{Z_{i}-Z_{a}^{*}}{Z_{i}+Z_{a}}
$$

is the (load dependent) reflection coefficient of the tag antenna-load system and

$$
S=\frac{1}{2 \eta}\left|\vec{E}_{\text {ind }}\right|^{2}
$$

assuming no polarization mismatch; $\eta$ denotes the impedance of the propagation medium ( $120 \pi$ in free space).

Nikitin et al. [9] exploited the Thevenin equivalent circuit of an antenna, carefully noting that such modeling assumes minimum scattering antennas. It can be easily shown that Thevenin equivalent circuit provides RCS equal to [6], [9]

$$
\sigma_{i}^{\text {thev }}=\frac{\lambda^{2} G^{2} R_{a}^{2}}{\pi\left|Z_{i}+Z_{a}\right|^{2}}
$$

or, equivalently from (2),

$$
K_{i}^{\text {thev }}=\frac{4 R_{a}^{2}}{\left|Z_{i}+Z_{a}\right|^{2}} .
$$

The authors in [9] denote differential backscattered power as $P_{\text {diff.bs }}=1 / 2\left|I_{1}-I_{2}\right|^{2} R_{a} G$, where $I_{i}$ is the current flowing at the Thevenin circuit when the antenna is connected to load $Z_{i}(i \in\{1,2\})$ and show that

$$
P_{\text {diff.bs }}=S \frac{\lambda^{2} G^{2}}{4 \pi}\left|\Gamma_{1}-\Gamma_{2}\right|^{2} .
$$

The ratio $P_{\text {diff.bs }} / S$ is coined in [9] as differential RCS $\Delta \sigma$. Thus, the system designer should maximize $\left|\Gamma_{1}-\Gamma_{2}\right|$, when the tag is connected to a minimum scattering antenna. This work studies what is needed to be maximized (or minimized) for efficient tag-to-reader communication, employing detection theory and no Thevenin modeling (and thus, applies to any tag antenna, including minimum scattering antennas as a special case); $1 / 2|I|^{2} R_{a} G$ is a valid representation of the backscattered power from tag to reader, only for minimum scattering antennas and not for the general tag antenna case: from Thevenin-produced equations (6) and (7), it can be seen that for open circuit load $Z_{i}=\infty$, the backscattered power from the tag is zero

$$
K^{\text {thev }}\left(Z_{i}=\infty\right)=\sigma^{\text {thev }}\left(Z_{i}=\infty\right)=0
$$

justifying the characterization minimum scattering antenna.

On the contrary, from (3), which applies to the general tag antenna case, it can be seen that RCS (and consecutively backscattered power from the tag to the reader) for the open circuit load is not zero

$$
\sigma\left(Z_{i}=\infty\right)=\frac{\lambda^{2}}{4 \pi} G^{2}\left|1-A_{s}\right|^{2} \neq 0 .
$$

The latter produces a zero power backscattered signal only for $A_{s}=1$. The antenna structural mode $A_{s}$ need not be unity, as shown in [10], where semipassive tags were studied, while $A_{s}$ was estimated using graphical methods. In this study, we provide closed-form $A_{s}$ calculation, while we study both passive, as well as semipassive, tags within more generalized context; our approach provides tag load selection constraints and rules without restricting discussion to specific tag/reader circuitry or minimum scattering antennas [11].

\section{TAG EFFICIENCY CONSTRAINTS}

From antenna scattering theory [8], [12], it is known that the scattered field from the tag back to the reader is given by

$$
\vec{E}_{i}=\vec{E}_{\mathrm{str}}-\vec{E}_{\mathrm{ant}}\left(Z_{i}, I_{a}\right) .
$$

The first term $\vec{E}_{\text {str }}$ corresponds to the load-independent tag antenna structural mode. The second term $\vec{E}_{\text {ant }}$ denotes the antenna mode and depends on the connected (to the antenna) load $Z_{i}$, as well as the current $I_{a}$ at the antenna terminals, at the absence of any external field. Specifically, the scattered field $\vec{E}_{i}$ at direction $(r, \theta, \phi)$ when the tag antenna is loaded with $Z_{i}$ can be expressed as [12], [13]

$$
\begin{aligned}
\vec{E}_{i} & \equiv \vec{E}_{i}(r, \theta, \phi)=\underbrace{I_{\mathrm{REF}} \frac{\vec{E}_{a}(r, \theta, \phi)}{I_{a}}}_{\vec{E}_{0}}\left(A_{s}-\Gamma_{i}\right) \\
& =\vec{E}_{0}(r, \theta, \phi)\left(A_{s}-\Gamma_{i}\right) \equiv \vec{E}_{0}\left(A_{s}-\Gamma_{i}\right) \\
& =\vec{E}_{0} \frac{2 \sqrt{\pi}}{\lambda G} \sqrt{\sigma_{i}} e^{+j \phi_{i}}
\end{aligned}
$$

where $I_{\mathrm{REF}}$ is the current induced by the incoming field at the tag antenna terminals, when the tag is terminated at load $Z_{a}^{*}$, and $\vec{E}_{a}$ is the field radiated by the tag antenna when the current at the tag antenna terminals is $I_{a}$ and no external incident wave is applied to the tag antenna. In (14), $\phi_{i}$ denotes the phase of the complex parameter $A_{s}-\Gamma_{i}$, while the amplitude of $A_{s}-\Gamma_{i}$ is set according to (3).

Notice that the term $\vec{E}_{a} / I_{a}$ is independent of $I_{a}$ and the term $\vec{E}_{0}$ is independent of the tag load $Z_{i}$. For example, assuming free-space propagation in a lossless medium, $\vec{E}_{a}(r, \theta, \phi) / I_{a}=$ $-j \eta 1 / 2 \lambda r \vec{\ell}_{e}(\theta, \phi) e^{-j \beta r}$, where $\vec{\ell}_{e}$ is the effective length of the receiving antenna at $(r, \theta, \phi)$ and $\beta=2 \pi / \lambda$. We will not necessarily assume free space in this study, but instead simply exploit the fact that $\vec{E}_{0}$ is tag load independent.

By definition, power $P_{i}(i=1$ or $i=2)$ of the backscattered field for the two different terminating loads $\left\{Z_{1}, Z_{2}\right\}$ is given by

$$
\frac{1}{2 \eta}\left|\vec{E}_{i}\right|^{2} \propto P_{i}=S \sigma_{i}
$$

assuming no losses due to polarization mismatch between tag and reader antennas. Notice that the power of the backscattered signal is not, in general, constant, but instead depends on the tag bit information ( $P_{1}=S \sigma_{1}$ for information bit "0" and $P_{2}=$ $S \sigma_{2}$ for information bit "1" in Fig. 2). Therefore, backscattered 


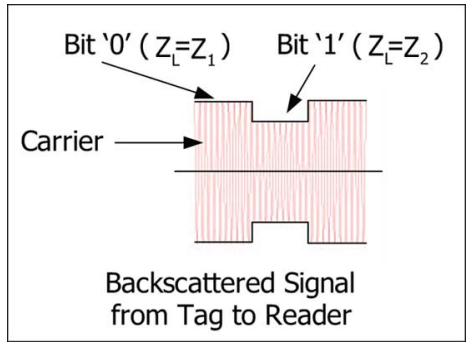

Fig. 2. Backscatter radio technique: signal backscattered from tag to reader binary-modulates information by changing the terminating $\operatorname{tag} \operatorname{load} Z_{L}$ between two states; each state corresponds to bit "0" or " 1. ."

signal power depends on the (in general, complex) reflection coefficient $\Gamma_{i}$ ( $\Gamma_{1}$ for bit " 0 " and $\Gamma_{2}$ for bit " 1 ").

\section{A. Maximum Backscattered Carrier Power Per Bit (Constraint 1)}

System designer should select loads $Z_{1}, Z_{2}$ such that backscattered signal power is maximized, regardless of the transmitted tag bits. In other words, the system designer should maximize average backscattered carrier power per bit $\left(P_{1}+P_{2}\right) / 2$, or equivalently, according to (15)

$$
\max \left\{\sigma_{1}+\sigma_{2}\right\} \text {. }
$$

This is a necessary condition that maximizes: 1) carrier signal, on top of which tag information will be modulated and 2) signal-to-noise ratio (SNR) at the reader's receiver that should exceed th e receiver's sensitivity for successful tag information extraction. In other words, neglecting the above constraint increases the chances for received power below reader's receiver sensitivity, and thus, no tag information reception. For semipassive tags, where in the forward link the received power is not exploited, but only decoded, the above constraint is important. Passive tags, which to date are forward-link limited, seem to be more lenient to the constraint [14]. However, the constraint will be valid, as the required power for their integrated circuits (ICs) is decreased with continued progress.

Notice that $\sigma_{i}$ also depends on the load-independent structural mode parameter $A_{s}$ of the tag antenna. Thus, the above maximization involves $A_{s}$. The Appendix provides a simple method for closed-form calculation of this parameter, for any tag antenna, given (measured or simulated) values of the tag's RCS.

\section{B. Minimum Bit-Error Rate (BER) Probability at the Reader (Constraint 2)}

Assuming that received signal power is above the receiver's sensitivity, the tag designer should also select terminating loads $Z_{i}(i=1$ or $i=2)$ at the tag, such that BER at the reader is minimized. The signal $y_{i}$, received at the reader antenna at point $(r, \theta, \phi)$, will be directly proportional to the backscattered field $\vec{E}_{i}$ at $(r, \theta, \phi)$, given by (14), plus additive receiver thermal noise

$$
y_{i}=h \underbrace{a \vec{E}_{i}}_{x_{i}}+n=h x_{i}+n
$$

where the complex parameter $h$ corresponds to the wireless channel (from tag to reader), $a$ accounts for the transform of a field quantity (e.g., in $\mathrm{V} / \mathrm{m}$ ) to a signal quantity (e.g., in $\mathrm{V}$ ), and finally, thermal noise $n$ is assumed a complex zero-mean circularly symmetric Gaussian random variable with expected power $\mathbb{E}\left\{|n|^{2}\right\}=\mathcal{N}_{0}$. Notice that for a given point $(r, \theta, \phi)$ at space, $a \vec{E}_{i}$ is a complex number.

The above flat fading model accounts for multipath, including both line-of-sight (LOS), as well as non-LOS tag-reader communication. No specific assumptions regarding the statistical distribution of $h$ is needed in our study. For cases where the tag-to-reader channel coherence bandwidth is smaller than the bit-rate (frequency selective nonflat wireless channel), deterministic modeling tools could be employed (e.g., ray tracing), which is beyond the scope of this paper. It is further noted that link budget analysis for the backscatter radio channel have recently started to appear in the literature (e.g., see [15] and the references therein).

Assuming knowledge of $a$ and $h$ (coherent receiver), maximum likelihood (ML) detection of $x_{1}=a \vec{E}_{1}$ for bit " 0 " or $x_{2}=a \vec{E}_{2}$ for bit " 1 ," in the presence of zero-mean additive complex circularly symmetric Gaussian noise, amounts to selecting bit " 0 " when the received signal $y$ is closer to $h x_{1}(\mid y-$ $\left.h x_{1}|<| y-h x_{2} \mid\right)$, or bit " 1 " when the received signal is closer to $h x_{2}\left(\left|y-h x_{1}\right|>\left|y-h x_{2}\right|\right)$. In other words, detection error $e$ is performed when the amount of noise exceeds half the distance between $h x_{1}$ and $h x_{2}$. The probability of such an event can be directly computed (e.g., see Appendix A in [16]), providing an expression of the BER to the reader

$$
\begin{aligned}
\operatorname{Pr}\{e\} & \triangleq Q\left(\frac{\left|h x_{1}-h x_{2}\right|}{2 \sqrt{\frac{\mathcal{N}_{0}}{2}}}\right) \\
& =Q\left(|h|\left|a \vec{E}_{0}\right| \frac{\left|A_{s}-\Gamma_{1}-A_{s}+\Gamma_{2}\right|}{2 \sqrt{\frac{\mathcal{N}_{0}}{2}}}\right) \\
& =Q\left(|h|\left|a \vec{E}_{0}\right| \frac{\left|\Gamma_{1}-\Gamma_{2}\right|}{2 \sqrt{\frac{\mathcal{N}_{0}}{2}}}\right)
\end{aligned}
$$

where $Q(x)=(1 / \sqrt{2 \pi}) \int_{x}^{+\infty} \exp \left(-x^{2} / 2\right) d x$ is the $Q$ function [17], which decreases with increasing $x$.

Therefore, BER minimization requires maximization of the reflection coefficient difference amplitude

$$
\max \left\{\left|\Gamma_{1}-\Gamma_{2}\right|\right\} .
$$

The above derivation is based on the ML (minimum distance) detector and applies to any binary modulation. That remark is important since environmental conditions, such as electromagnetic (EM) coupling at the vicinity of the tag or the reader, could alter the transmitted signals. A similar result, involving received 
voltage and the complementary error function has been reported in [12] for the specific case of amplitude-shift keying (ASK) and phase-shift keying (PSK) modulation (used in EPC C1G2) and the receiver from [11] or nonideal binary modulation in [18]. The result of (21) agrees with [9], where Thevenin-equivalent circuit and minimum scattering antennas were assumed. Notice that the above derivation has employed simple detection theory, without any type of Thevenin-based antenna-tag chip modeling or any prior assumption regarding the tag antenna or the tag circuitry.

It is further noted that

$$
\begin{aligned}
\operatorname{Pr}\{e\} & \triangleq Q\left(\frac{\left|h x_{1}-h x_{2}\right|}{2 \sqrt{\frac{\mathcal{N}_{0}}{2}}}\right) \\
& =Q\left(|h|\left|a \vec{E}_{0}\right| \frac{\left|\Gamma_{1}-\Gamma_{2}\right|}{2 \sqrt{\frac{\mathcal{N}_{0}}{2}}}\right) \\
& =Q\left(\frac{|h| \sqrt{\left|x_{1}\right|^{2}+\left|x_{2}\right|^{2}-2 \operatorname{Real}\left\{x_{1} x_{2}^{*}\right\}}}{2 \sqrt{\frac{\mathcal{N}_{0}}{2}}}\right) \\
& =Q\left(|h| \frac{\sqrt{\pi}}{\lambda G}\left|a \vec{E}_{0}\right| \frac{\sqrt{\sigma_{1}+\sigma_{2}-2 \sqrt{\sigma_{1}} \sqrt{\sigma_{2}} \cos (\delta \phi)}}{\sqrt{\frac{\mathcal{N}_{0}}{2}}}\right)
\end{aligned}
$$

where $\delta \phi=\phi_{1}-\phi_{2}$ is the phase difference of the backscattered (from the tag) binary signals, according to (14) and definition of $x_{i}$ in (17).

For PSK modulation, $\cos \left(\phi_{1}-\phi_{2}\right)=-1$. Passive tags can have $\left|\Gamma_{1}\right| /\left|\Gamma_{2}\right|=\kappa \leq 1$ and $\left|\Gamma_{1}-\Gamma_{2}\right| \leq 1+\kappa \leq 2$ (notice that for $\Gamma_{2}=1$ in this case, $\left.\left|\Gamma_{1}-\Gamma_{2}\right|=1+\kappa\right)$. Semipassive (battery assisted) tags can have $\left|\Gamma_{1}\right| /\left|\Gamma_{2}\right|=1$ and $\left|\Gamma_{1}-\Gamma_{2}\right| \leq 2$ with $\left|\Gamma_{1}-\Gamma_{2}\right|=2$ the preferred value.

Similarly, for ASK modulation, $\cos \left(\phi_{1}-\phi_{2}\right)=+1$. For passive tags, $\Gamma_{1}=0$ and $\left|\Gamma_{1}-\Gamma_{2}\right| \leq 1$. For semipassive tags and on-off keying $(\mathrm{OOK}), \Gamma_{1}=A_{s}\left(\sigma_{1}=0\right)$ and $\left|\Gamma_{1}-\Gamma_{2}\right|=$ $(1+\kappa)\left|\Gamma_{2}\right| \leq 1+\kappa$. Notice that, in this case of OOK, the value of $\Gamma_{2}$ that maximizes $\left|\Gamma_{1}-\Gamma_{2}\right|=1+\kappa$ should have amplitude $\left|\Gamma_{2}\right|=1$ and phase difference $\pi$ compared to phase of $\Gamma_{1}$. In other words, $\cos \left(\theta_{1}-\theta_{2}\right)=-1 \neq \cos \left(\phi_{1}-\phi_{2}\right)$, where $\theta_{i}$ is the phase of $\Gamma_{i}$. That means that the phase difference of reflection coefficients does not necessarily define the modulation type.

Furthermore, the above example shows that careful selection of the modulation and respective minimization of reader BER could employ estimation of the tag antenna structural mode $A_{s}$ ( $\Gamma_{1}=A_{s}$ for the above OOK example), which is in sharp contrast to what is commonly believed in the field. The Appendix provides a method for $A_{s}$ estimation for any tag antenna type.

Summarizing for a (nonideal) pseudo-PSK (or ASK) modulation (studied in [19]), the following inequality holds:

$$
0 \leq\left|\Gamma_{1}-\Gamma_{2}\right| \leq 1+\kappa \leq 2 .
$$

Finally, maximization of the load-independent antenna-specific term $\left|\vec{E}_{0}\right|$ is also needed for reduced BER. Additionally, maximization of $\sigma_{1}+\sigma_{2}$ in (16) requires consideration of the antenna-specific structural mode parameter $A_{s}$, already discussed in Section III-A. Thus, maximization of $\left|\Gamma_{1}-\Gamma_{2}\right|$, even though necessary, is clearly not sufficient for improved backscatter tag-to-reader communication.

\section{Note on Tag Efficiency Variance}

We have already noted that backscattered power is not, in general, constant, but instead depends on tag bit information. If backscattered field power $P_{\text {out }}$ varies between $P_{1}$ and $P_{2}$, then tag efficiency $K$ also varies for given induced reader field density $S$ at the tag [according to (2) and (15)]

$$
\operatorname{var}(K)=\frac{16 \pi^{2}}{\lambda^{4} G^{4} S^{2}} \operatorname{var}\left(P_{\text {out }}\right) \propto \operatorname{var}\left(P_{\text {out }}\right)
$$

where $\operatorname{var}(x)$ denotes the statistical variance of $x$.

For $N$ consecutive backscattered bits from tag to reader, the average backscattered power is given (for large $N$ according to the law of large numbers) by

$$
P_{\text {out }}(N)=\frac{\mu P_{1}+(N-\mu) P_{2}}{N}=\frac{P_{1}-P_{2}}{N} \mu+P_{2}
$$

where $\mu$ is binomially distributed

$$
\operatorname{Pr}\{\mu\}=\left(\begin{array}{l}
N \\
\mu
\end{array}\right)\left(\frac{1}{2}\right)^{\mu}\left(\frac{1}{2}\right)^{N-\mu}=\left(\begin{array}{l}
N \\
\mu
\end{array}\right)\left(\frac{1}{2}\right)^{N} .
$$

Thus,

$$
\begin{aligned}
\operatorname{var}\left(P_{\text {out }}(N)\right) & =\left(\frac{P_{1}-P_{2}}{N}\right)^{2} \operatorname{var}(\mu) \\
& =\frac{\left(P_{1}-P_{2}\right)^{2}}{4 N} \\
& =\frac{S^{2}\left(\sigma_{1}-\sigma_{2}\right)^{2}}{4 N}
\end{aligned}
$$

where we have used (15) and the fact that $\operatorname{var}(\mu)=N / 4$, according to (27). Exploiting (29) in(25), we get

$$
\operatorname{var}(K)=\frac{4 \pi^{2}}{\lambda^{4} G^{4}} \frac{\left(\sigma_{1}-\sigma_{2}\right)^{2}}{N} \propto \frac{\left(\sigma_{1}-\sigma_{2}\right)^{2}}{N} .
$$

For example, for the 911-MHz passive tag antenna proposed in $[20]$ and $N=96$, tag efficiency variance ${ }^{1}$ amounts to $\operatorname{var}(K)=0.0033=(0.057)^{2}$. System engineers should take into account its standard deviation and worst case analysis should be performed. Future work should further study tag efficiency variance as a function of specific reader implementations and backscatter radio protocols.

\section{EfFicient Tags: Case Study}

Passive (battery-less) tags usually employ, apart from the tag antenna, digital logic in the form of a low power microcontroller or an application-specific IC. Such logic is designed to draw a

\footnotetext{
${ }^{1}$ EPC class 1 generation 2 (EPC Gen 2 for brevity) employs at least 96 bits for any tag-transmitted ID $(N \geq 96)$. A Gen 2 standard is available online at http://www.epcglobalinc.org/standards/uhfc1g2
} 


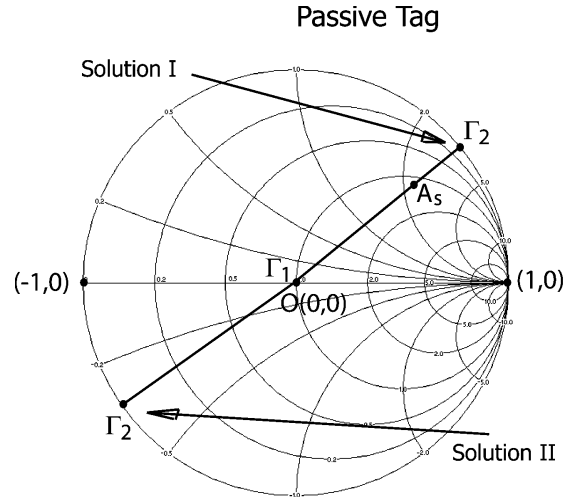

(a)

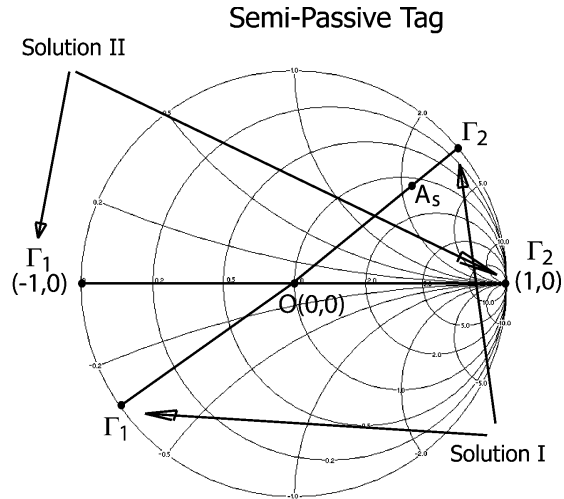

(b)

Fig. 3. Selection of tag load and corresponding reflection coefficients $\Gamma_{1}, \Gamma_{2}$ for given tag antenna structural mode $A_{s}$. The depicted selections minimize reader detection probability, but provide different average backscattered carrier power per bit. Both passive and semipassive tags are considered (a) Passive tag. (b) Semipassive tag.

small amount of current (low power) and is usually modeled by a high resistance connected in parallel to a capacitive load (or equivalently, a small resistive load in series with a capacitive load) (e.g., [9] and [21]). A voltage multiplier and regulator is also needed to power the tag logic (chip), as well as the backscattered field modulator that changes the overall impedance, seen by the tag antenna, between two loads $\left(Z_{1}\right.$ and $Z_{2}$ in Fig. 1). Given that the tag antenna has, in general, different equivalent impedance than the chip, a matching network between the antenna and chip is utilized.

This is the approach followed in this study for the case of passive tags. Depending on the selected load, the modulation can change the amplitude of the backscattered field (usually by changing the real part of the load and corresponding to ASK modulation) or the phase of the backscattered field (usually by changing the imaginary part of the load and corresponding to PSK modulation).

Alternatively, the passive-tag designer could trade the amount of power scattered back (to the reader) with power delivered to the tag chip, using imperfect matching (e.g., see discussion in [22] or in [14, Ch. 5]).

In the following, we provide general tag load selection rules for both passive, as well as semipassive tags, without restricting discussion to specific tag designs or minimum-scattering antennas only (we assume, in general, that $A_{s} \neq 1$ ).

\section{A. Passive Tags}

For batteryless passive tags, tag load $Z_{1}$ usually corresponds to perfect match $\left(\Gamma_{1}=0\right)$. In that way, power transfer from the tag antenna to tag chip is maintained, at least for the duration of bit " 0 ." The tag load $Z_{2}$ (and corresponding $\Gamma_{2}$ ) is selected such that $\Gamma_{2}-A_{s}$ is collinear with $\Gamma_{1}-A_{s}$. Two possible solutions for $\Gamma_{2}$ on the unit circle are depicted: solution I and II (Fig. 3, left). Both satisfy constraint 2 of (21), while solution II provides for higher average backscatter carrier power per bit (constraint 1) than solution I since $\left|\Gamma_{2}-A_{s}\right|$ is maximized. For the special case of $A_{s}=1$, one solution could be $\Gamma_{2}=A_{s}=1$. A better solution with higher backscattered carrier power per bit would be $\Gamma_{2}=-1$.

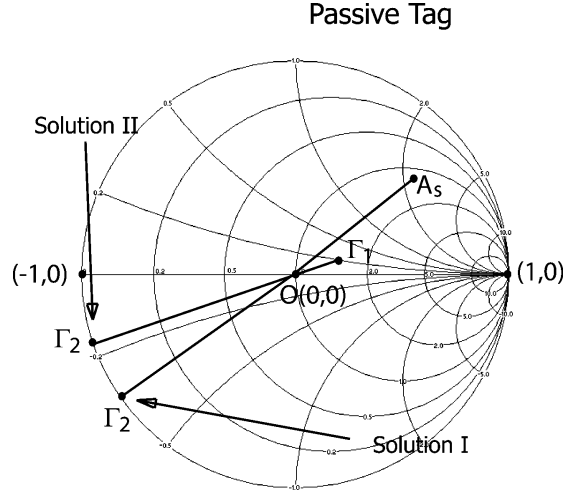

Fig. 4. Selection of tag load for the case of passive tag with imperfect matching $\left(\Gamma_{1} \neq 0\right)$. Depicted solutions I and II satisfy constraints 1 and 2, respectively.

An alternative approach (for the general case of $A_{s} \neq 1$ ) uses $\Gamma_{2}=A_{s}$. In that way, tag efficiency variance proportional to $\sigma_{1}-\sigma_{2}$ is slightly reduced at the expense of higher BER at the reader (since $\left|\Gamma_{1}-\Gamma_{2}\right|$ is also reduced, compared to solution II depicted in Fig. 3, left).

Fig. 4 depicts the case where perfect matching for load $Z_{1}$ cannot be achieved, either due to parasitic elements on the printed circuit board and/or the fact that tags operate in a range of carrier frequencies (and not a single carrier frequency). The depicted reflection coefficient $\Gamma_{1}$ corresponds to a voltage standing-wave ratio (VSWR) on the order of 1.5:1. Depicted solution I for $\Gamma_{2}$ (and respective load $Z_{2}$ ) maximizes average backscattered carrier per bit (16) while achieving near-maximum $\left|\Gamma_{1}-\Gamma_{2}\right|$; solution II maximizes $\left|\Gamma_{1}-\Gamma_{2}\right|$ (21) while achieving near-maximum average backscattered carrier per bit. A system designer could select either solutions or a reflection coefficient (and respective load) on the unit circle and between the two aforementioned points.

In any case of matching (perfect or imperfect), it becomes clear that passive tag design for improved tag-to-reader backscatter communication requires consideration of parameter $A_{s}$, which is an antenna-specific load-independent parameter. Its calculation is provided in the Appendix. 
A first example can be given for a planar tag, operating at $915 \mathrm{MHz}$ with $Z_{a}=6.7+j 148.8$. Given RCS values for different loads, the Appendix method calculates structural mode parameter $A_{s}=0.6047+j 0.5042=0.7873<39.82^{\circ}$. Solution I with $\Gamma_{2}=1<39.82^{\circ}$ provides $\sigma_{2}^{\mathrm{I}}=0.0011 \mathrm{~m}^{2}$. Solution II with $\Gamma_{2}=1<(180+39.82)^{\circ}$ achieves $\sigma_{2}^{\mathrm{II}}=$ $0.0763 \mathrm{~m}^{2}$. Thus, the ratio $\left(\sigma_{2}^{\mathrm{II}}+\sigma_{1}\right) /\left(\sigma_{2}^{\mathrm{I}}+\sigma_{1}\right)=5.73$ with $\sigma_{1}$ the RCS for zero reflection coefficient $\Gamma_{1}=0$ (matched load). The observed ratio corresponds to gain of $7.58 \mathrm{~dB}$ in terms of received average carrier power per bit that could be effectively translated in an improved link budget. For example, when solution I provides a link budget that operates the reader $\sim 7 \mathrm{~dB}$ below its minimum received power (sensitivity) threshold, solution II can provide reception with the BER given by (20).

A second example contains the case of a passive tag antenna fabricated with a thin copper layer on PET $\left(\epsilon_{r}=3.2\right)$ at $911 \mathrm{MHz}$ with $Z_{a}=2+j 146$ and near-isotropic RCS [20]. For matched load $\left(\Gamma_{1}=0\right)$, RCS amounts to $\sigma_{1}=0.0044 \mathrm{~m}^{2}$, and for $\Gamma=-1$, RCS amounts to $0.0141 \mathrm{~m}^{2}$. Since from [20] only two different RCS values are provided, the structural mode was found to be $A_{s}=0.0062 \pm j 0.7114$, assuming uniform antenna gain $(G=1)$. Consecutively, $\Gamma_{2}^{\mathrm{I}}=0.0868 \pm j 0.9962$ with $\sigma_{2}^{\mathrm{I}}=0.0007 \mathrm{~m}^{2}$ and $\Gamma_{2}^{\mathrm{II}}=-0.0868 \mp j 0.9962$ with $\sigma_{2}^{\mathrm{II}}=0.0254 \mathrm{~m}^{2}$. Thus, the ratio $\left(\sigma_{2}^{\mathrm{II}}+\sigma_{1}\right) /\left(\sigma_{2}^{\mathrm{I}}+\sigma_{1}\right)=5.84$, resulting to a power gain of $7.66 \mathrm{~dB}$, which effectively improves link budget.

\section{B. Semipassive (Battery-Assisted) Tags}

For semipassive tags, the available battery powers the tag chip during transmission of information from the tag to the reader. The battery is used to energize the tag chip and it is not used for any type of signal conditioning such as signal amplification, filtering, etc. Therefore, the mechanism for tag-to-reader communication is through backscattering, and hence, the term semipassive. Thus, semipassive tags do not require power scavenging and relevant voltage regulators.

Given that power transfer from reader (or tag antenna) to the tag chip is not needed during tag-to-reader communication, $\Gamma_{1}$ need not be equal to zero, while both reflection coefficients should be on the unit circle (in order to maximize $\left|\Gamma_{1}-\Gamma_{2}\right|$ ). Fig. 3, right, depicts two solutions that both achieve the maximum $\left|\Gamma_{1}-\Gamma_{2}\right|=2$. In general, solution II could represent any two diametrically opposite points on the unit circle.

Both Solutions I and II in Fig. 3, right, as well as any two diametrically opposite points on the unit circle achieve the same $\sigma_{1}+\sigma_{2}$ [constraint 1 of (16)]. The proof follows: denote $\Gamma_{1}=$ $a+j b$, diametrically opposite $\Gamma_{2}=-a-j b$, both on the unit circle $|a|^{2}+|b|^{2}=1$ and $A_{s}=x+j y$

$$
\begin{aligned}
\sigma_{1}+\sigma_{2} \propto & \left|\Gamma_{1}-A_{s}\right|^{2}+\left|\Gamma_{2}-A_{s}\right|^{2} \\
= & |(a-x)+j(b-y)|^{2} \\
& +|-(a+x)-j(b+y)|^{2} \\
= & 2+2\left|A_{s}\right|^{2} .
\end{aligned}
$$

In other words, solutions I and II of Fig. 3, right, as well as any diametrically opposite $\left(\Gamma_{1}, \Gamma_{2}\right)$ on the unit circle maximize $\left|\Gamma_{1}-\Gamma_{2}\right|$ (constraint 2) and achieve the same sum in (16) (constraint 1). In order to maximize the sum of constraint 1 , the tag antenna-specific parameter $\left|A_{s}\right|$ must be maximized, i.e., the tag antenna should be carefully designed.

For the special case of $A_{s}=1$, one possible solution is given by $\Gamma_{1}=A_{s}=1, \Gamma_{2}=-1$. From the above, it is seen that the solution $\Gamma_{1}=j, \Gamma_{2}=-j$ provides the same results in terms of constraints 1 and 2 (for $A_{s}=1$ ), while achieving zero tag efficiency variance [see (30)].

An alternative approach (for the general case of $A_{s} \neq 1$ ) is to select $\Gamma_{2}=A_{s}$ and $\Gamma_{1}$ on the unit circle, diametrically opposite to $\overrightarrow{O A_{s}}$. Such a selection clearly provides higher detection error probability (since $\left|\Gamma_{1}-\Gamma_{2}\right|$ is reduced), while providing smaller tag efficiency variance (compared to solution I of Fig. 3, right).

\section{CONCLUSION}

Load-independent antenna parameters should be considered for improved tag-to-reader communication, contrary to what is commonly believed in the field. We provided simple rules on selecting the terminating loads for efficient tag-to-reader backscatter communication. Derivation employed basic communication theory without restricting discussion to minimum scattering antennas. It was shown that maximization of reflection coefficient difference amplitude $\left|\Gamma_{1}-\Gamma_{2}\right|$ is not sufficient since additional constraints exist, while antennas are not necessarily minimum scattering (structural mode $A_{s} \neq 1$ ). A methodology was provided to select the tag loads for both passive, as well as semipassive, tags based on the tag antenna structural mode. A simple closed-form calculation of the structural mode for any tag antenna was also given.

\section{APPENDIX \\ Closed-Form Calculation of Tag Antenna STRUCTURAL MODE}

In order to calculate the structural mode parameter $A_{s}$, three values $\sigma_{1}, \sigma_{2}, \sigma_{3}$ of the antenna RCS are needed, corresponding to three different loads $Z_{1}, Z_{2}, Z_{3}$ (or equivalently, reflection coefficients $\left.\Gamma_{1}, \Gamma_{2}, \Gamma_{3}\right)$. Antenna RCS can be measured experimentally [6] or estimated through simulation. Denote complex $\Gamma_{i}=x_{i}+j y_{i}$ that corresponds to load $Z_{i}$ and RCS $\sigma_{i}$ with $i \in\{1,2,3\}$ and the unknown $A_{s}=x+j y$. From (3), we have three circle equations

$$
\begin{aligned}
& \left(x-x_{1}\right)^{2}+\left(y-y_{1}\right)^{2}=\frac{4 \pi}{\lambda^{2} G^{2}} \sigma_{1} \\
& \left(x-x_{2}\right)^{2}+\left(y-y_{2}\right)^{2}=\frac{4 \pi}{\lambda^{2} G^{2}} \sigma_{2} \\
& \left(x-x_{3}\right)^{2}+\left(y-y_{3}\right)^{2}=\frac{4 \pi}{\lambda^{2} G^{2}} \sigma_{3} .
\end{aligned}
$$

Dividing (A.1) by (A.2) and setting $\kappa_{12}=\sigma_{1} / \sigma_{2}$, we get a circle equation centered at $\left(x_{1}^{*}, y_{1}^{*}\right)$ and radius $r_{1}$

$$
\begin{aligned}
\left(x_{1}^{*}, y_{1}^{*}\right)= & \left(\frac{x_{1}-\kappa_{12} x_{2}}{1-\kappa_{12}}, \frac{y_{1}-\kappa_{12} y_{2}}{1-\kappa_{12}}\right) \\
r_{1}^{2}= & \left(\frac{x_{1}-\kappa_{12} x_{2}}{1-\kappa_{12}}\right)^{2}+\left(\frac{y_{1}-\kappa_{12} y_{2}}{1-\kappa_{12}}\right)^{2} \\
& +\frac{\kappa_{12}\left(x_{2}^{2}+y_{2}^{2}\right)}{1-\kappa_{12}}-\frac{x_{1}^{2}+y_{1}^{2}}{1-\kappa_{12}},\left(x-x_{1}^{*}\right)^{2} \\
& +\left(y-y_{1}^{*}\right)^{2}=r_{1}^{2} .
\end{aligned}
$$


Dividing (A.1) by (A.3) and setting $\kappa_{13}=\sigma_{1} / \sigma_{3}$, we get a circle equation centered at $\left(x_{2}^{*}, y_{2}^{*}\right)$ and radius $r_{2}$

$$
\begin{aligned}
\left(x_{2}^{*}, y_{2}^{*}\right)= & \left(\frac{x_{1}-\kappa_{13} x_{3}}{1-\kappa_{13}}, \frac{y_{1}-\kappa_{13} y_{3}}{1-\kappa_{13}}\right) \\
r_{2}^{2}= & \left(\frac{x_{1}-\kappa_{13} x_{3}}{1-\kappa_{13}}\right)^{2}+\left(\frac{y_{1}-\kappa_{13} y_{3}}{1-\kappa_{13}}\right)^{2} \\
& +\frac{\kappa_{13}\left(x_{3}^{2}+y_{3}^{2}\right)}{1-\kappa_{13}}-\frac{x_{1}^{2}+y_{1}^{2}}{1-\kappa_{13}},\left(x-x_{2}^{*}\right)^{2} \\
& +\left(y-y_{2}^{*}\right)^{2} \\
= & r_{2}^{2} .
\end{aligned}
$$

Finally, dividing (A.2) by (A.3) and setting $\kappa_{23}=\sigma_{2} / \sigma_{3}$, we get a circle equation centered at $\left(x_{3}^{*}, y_{3}^{*}\right)$ and radius $r_{3}$

$$
\begin{aligned}
\left(x_{3}^{*}, y_{3}^{*}\right)= & \left(\frac{x_{2}-\kappa_{23} x_{3}}{1-\kappa_{23}}, \frac{y_{2}-\kappa_{23} y_{3}}{1-\kappa_{23}}\right) \\
r_{3}^{2}= & \left(\frac{x_{2}-\kappa_{23} x_{3}}{1-\kappa_{23}}\right)^{2}+\left(\frac{y_{2}-\kappa_{23} y_{3}}{1-\kappa_{23}}\right)^{2} \\
& +\frac{\kappa_{23}\left(x_{3}^{2}+y_{3}^{2}\right)}{1-\kappa_{23}}-\frac{x_{2}^{2}+y_{2}^{2}}{1-\kappa_{23}},\left(x-x_{3}^{*}\right)^{2} \\
& +\left(y-y_{3}^{*}\right)^{2} \\
= & r_{3}^{2} .
\end{aligned}
$$

If a solution $(x, y)$ exists, that will be the intersection of circles of (A.4) and (A.5) that validates circle of (A.6)

$$
\begin{aligned}
(x, y)=\left(x_{1}^{*}+\frac{a}{d}\left(x_{2}^{*}-x_{1}^{*}\right)+\frac{h}{d}\left(y_{2}^{*}-y_{1}^{*}\right),\right. \\
\left.y_{1}^{*}+\frac{a}{d}\left(y_{2}^{*}-y_{1}^{*}\right)-\frac{h}{d}\left(x_{2}^{*}-x_{1}^{*}\right)\right)
\end{aligned}
$$

or

$$
\begin{aligned}
&(x, y)=\left(x_{1}^{*}+\frac{a}{d}\left(x_{2}^{*}-x_{1}^{*}\right)-\frac{h}{d}\left(y_{2}^{*}-y_{1}^{*}\right),\right. \\
&\left.y_{1}^{*}+\frac{a}{d}\left(y_{2}^{*}-y_{1}^{*}\right)+\frac{h}{d}\left(x_{2}^{*}-x_{1}^{*}\right)\right)
\end{aligned}
$$

where

$$
\begin{aligned}
& d=\sqrt{\left(x_{1}^{*}-x_{2}^{*}\right)^{2}+\left(y_{1}^{*}-y_{2}^{*}\right)^{2}} \\
& a=\frac{r_{1}^{2}-r_{2}^{2}+d^{2}}{2 d} \quad h=\sqrt{r_{1}^{2}-a^{2}} .
\end{aligned}
$$

The pair $(x, y)$ above that validates (A.6) provides the unknown $A_{s}=x+j y$.

As an example, consider the case of a planar tag antenna at $915 \mathrm{MHz}$ and $Z_{a}=6.7+148.8 j, \Gamma_{1}=1$ (open circuit) with $\sigma_{1}=0.0098 \mathrm{~m}^{2}, \Gamma_{2}=0$ (matched load) with $\sigma_{2}=0.0148 \mathrm{~m}^{2}$ and $\Gamma_{3}=j$ (reactive load) with $\sigma_{3}=0.0146 \mathrm{~m}^{2}$ [10]. The result of the above calculation provides an exact value of $A_{s}=$ $0.6047+0.5042 j$. That is close to the approximate value provided in [10], where $A_{s}$ is graphically estimated on a Smith chart.

\section{REFERENCES}

[1] H. Stockman, "Communication by means of reflected power," Proc. IRE, vol. 36, no. 10, pp. 1196-1204, Oct. 1948.
[2] J. Paradiso, L. Pardue, K. Hsiao, and A. Benbasat, "Electromagnetic tagging for electronic music interfaces," J. New Music Res., vol. 32, no. 4, pp. 395-409, Dec. 2003.

[3] G. Vannucci, A. Bletsas, and D. Leigh, "A software-defined radio system for backscatter sensor networks," IEEE Trans. Wireless Commun., vol. 7, no. 6, pp. 2170-2179, Jun. 2008.

[4] A. Bletsas, S. Siachalou, and J. N. Sahalos, "Anti-collision tags for backscatter sensor networks," in 38th Eur. Microw. Conf., Amsterdam, The Netherlands, Oct. 2008, pp. 179-182.

[5] A. Bletsas, S. Siachalou, and J. N. Sahalos, "Anti-collision backscatter sensor networks," IEEE Trans. Wireless Commun., vol. 8, no. 10, pp. 5018-5029, Oct. 2009.

[6] P. V. Nikitin and K. V. S. Rao, "Theory and measurement of backscattering from RFID tags," IEEE Antennas Propag. Mag., vol. 48, no. 6, pp. 212-218, Dec. 2006.

[7] R. B. Green, "The general theory of antenna scattering," Ph.D. dissertation, Dept. Elect. Comput. Eng., Ohio State Univ, Columbus, OH, 1963.

[8] C. A. Balanis, Antenna Theory: Analysis and Design, 3rd ed. Hoboken, NJ: Wiley, 2005.

[9] P. V. Nikitin, K. V. S. Rao, and R. Martinez, "Differential RCS of RFID tag," Electron. Lett., vol. 43, no. 8, pp. 431-432, Apr. 2007.

[10] C.-C. Yen, A. E. Gutierrez, D. Veeramani, and D. van der Weide, "Radar cross-section analysis of backscattering RFID tags," IEEE Antennas Wireless Propag. Lett., vol. 6, pp. 279-281, 2007.

[11] G. De Vita and G. Iannaccone, "Design criteria for the RF section of UHF and microwave passive RFID transponders," IEEE Trans. Microw. Theory Tech., vol. 53, no. 9, pp. 2978-2990, Sep. 2005.

[12] F. Fuschini, C. Piersanti, F. Paolazzi, and G. Falciasecca, "Analytical approach to the backscattering from UHF RFID transpoder," IEEE Antennas Wireless Propag. Lett., vol. 7, pp. 33-35, 2008.

[13] R. C. Hansen, "Relationships between antennas as scatterers and as radiators," Proc. IEEE, vol. 77, no. 5, pp. 659-662, May 1989.

[14] D. M. Dobkin, The RF in RFID: Passive UHF RFID in Practice. Burlington, MA: Newnes, 2008.

[15] J. D. Griffin and G. D. Durgin, "Complete link budgets for backscatterradio and RFID systems," IEEE Antennas Propag. Mag., vol. 51, no. 2, pp. 11-25, Apr. 2009

[16] D. Tse and P. Viswanath, Fundamentals of Wireless Communication. Cambridge, U.K.: Cambridge Univ. Press, 2005.

[17] J. G. Proakis, Digital Communications, 4th ed. New York: McGrawHill, 2001.

[18] F. Fuschini, C. Piersanti, F. Paolazzi, and G. Falciasecca, "On the efficiency of load modulation in RFID systems operating in real environment," IEEE Antennas Wireless Propag. Lett., vol. 7, pp. 243-246, 2008.

[19] J.-P. Curty, M. Declerq, C. Dehollain, and N. Joehl, Design and Optimization of Passive UHF RFIF Systems. Berlin, Germany: Springer, 2007.

[20] J. Ahn, H. Jang, H. Moon, J.-W. Lee, and B. Lee, "Inductively coupled compact RFID tag antenna at $910 \mathrm{MHz}$ with near-isotropic radar crosssection (RCS) patterns," IEEE Antennas Wireless Propag. Lett., vol. 6, pp. 518-520, 2007.

[21] J.-P. Curty, N. Joehl, C. Dehollain, and M. J. Declercq, "Remotely powered addressable UHF RFID integrated system," IEEE J. Solid-State Circuits, vol. 40, no. 11, pp. 2193-2202, Nov. 2005.

[22] U. Karthaus and M. Fischer, "Fully integrated passive UHF RFID transponder IC with $16.7 \mu \mathrm{W}$ minimum RF input power," IEEE $J$. Solid-State Circuits, vol. 38, no. 10, pp. 1602-1608, Oct. 2003.

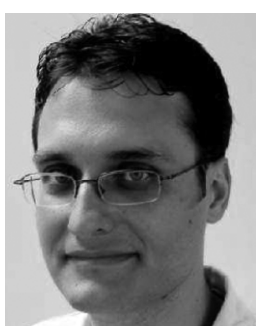

Aggelos Bletsas (S'03-M'05) received the Diploma degree in electrical and computer engineering (with excellence) from the Aristotle University of Thessaloniki, Thessaloniki, Greece, in 1998, and the S.M. and Ph.D. degrees from the Massachusetts Institute of Technology (MIT), Cambridge, in 2001 and 2005, respectively.

He was with Mitsubishi Electric Research Laboratories (MERL), Cambridge MA, as a Postdoctoral Fellow and with the Radiocommunications Laboratory (RCL), Department of Physics, Aristotle University of Thessaloniki, as a Visiting Scientist. In Summer 2009, he joined the Electronic and Computer Engineering Department, Technical University of Crete, as an Assistant Professor. His research interests focus on scalable wireless communication and networking with an emphasis on relay techniques, 
signal processing for communication, radio hardware/software implementations for wireless transceivers and low-cost backscatter sensor networks, RFID, time/frequency metrology, and bibliometrics.

Dr. Bletsas was the recipient of the Best Undergraduate Thesis Award of Ericsson Hellas for the development of a complete text-to-speech system for the Greek language, commercialized in 1999. He was also the recipient of awards for undergraduate excellence of the Technical Chamber of Greece and State Scholarship Foundation (IKY). During his graduate studies at MIT, he was supported by fellowship awards from BT and Nortel Networks. He was the corecipient of the IEEE Communications Society 2008 Marconi Prize Paper Award in wireless communications and Best Paper Distinction of the IEEE ISWCS 2009, Siena, Italy.

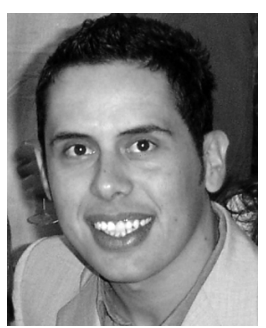

Anton G. Dimitriou (S'01-M'07) was born in Ierapetra, Greece, in 1977. He received the Diploma and $\mathrm{Ph} . \mathrm{D}$. degrees in electrical and computer engineering from the Aristotle University of Thessaloniki (AUTH), Thessaloniki, Greece, in 2001, and 2006 respectively.

Since 2007, he was with the Department of Electrical and Computer Engineering, AUTH. Since 2001, he has participated in 16 research projects in the fields of communications, antennas, propagation, signal processing, and RFIDs, including the design of a global system for mobile communications (GSM) cellular network inside the Olympic stadium for the 2004 Olympic Games, a prototype two-way translation system between sign language and speech, and an RFID pilot network inside a hospital. He has authored or coauthored over 20 journal and conference papers. His current research interests are in the areas of EM-wave propagation, planning and optimization of wireless networks, and RFIDs.

Dr. Dimitriou is a member of the Technical Chamber of Greece. He was the recipient of the Ericsson Award of Excellence in Telecommunications for the best undergraduate thesis in 2001.

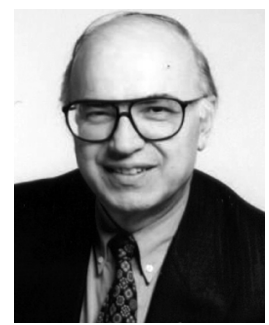

John N. Sahalos (M'75-SM'84-F'06-LF'10) received the B.Sc. and Ph.D. degrees in physics from the Aristotle University of Thessaloniki (AUTH), Thessaloniki, Greece, in 1967 and 1974, respectively, the Diploma (BCE + MCE) degree in civil engineering from the School of Engineering, AUTH, in 1975, and the Professional Diploma of postgraduate studies in electronic physics from the School of Science, AUTH, in 1975.

From 1971 to 1974, he was a Teaching Assistant with the Department of Physics, AUTH, and rom 1974 to 1976, he was an Instructor. In 1976, he was with the ElectroScience Laboratory, The Ohio State University, Columbus, as a Postdoctoral University Fellow. From 1977 to 1986, he was a Professor with the Electrical Engineering Department, University of Thrace, Thrace, Greece, and Director of the Microwaves Laboratory. Since 1986, he has been a Professor with the School of Science, AUTH, where he is also the Director of postgraduate studies in electronic physics and the Director of the Radio-Communications Laboratory (RCL). From 1981 to 1982, he was a Visiting Professor with the Department of Electrical and Computer Engineering, University of Colorado at Boulder From 1989 to 1990, he was a Visiting Professor with the Technical University of Madrid, Madrid, Spain. He is a consultant in industry. He has authored three books in Greek and seven book chapters. He has authored or coauthored over 300 papers appearing in scientific literature. He also authored The Orthogonal Methods of Array Synthesis, Theory and the ORAMA Computer Tool (Wiley, 2006). He has been of the Editorial Board of three scientific journals. His research interests are in the areas of antennas, high-frequency techniques, communications, electromagnetic compatibility (EMC)/electromagnetic interference (EMI), microwaves, and biomedical engineering.

Dr. Sahalos is a Professional Engineer. From 2002 to 2004, he was of the Board of Directors of the OTE, the largest Telecommunications Company in Southeast Europe. He has been a technical advisor for several national and international committees, as well as for several mobile communications companies. Since 1992, he has been a member of Commissions A and E, URSI. Since 1998, he has been the president of the Greek committees, URSI. From 2004 to 2008, he was the president of the section of Informatics, Telecommunications and Systems, National Committee of Research and Technology. He is an honorary member of the Radio-electrology Society. He is a member of the Greek Physical Society and the Technical Chamber of Greece. He is the creator and leader of an EMC network with five laboratories (three from the academy and two from the industry). He has been honored with a special investigation fellowship of the Ministry of Education and Science, Spain. He also has been honored from several institutes and organizations. He was elected by the department representatives of the AUTH as the vice-chairman of the Research Committee of AUTH (2007-2010). 